\title{
Die 58. Internationale Mathematik-Olympiade
}

\author{
Jürgen Prestin
}

\begin{abstract}
Die 58. Internationale Mathematik-Olympiade fand vom 12. bis 23. Juli 2017 in Rio de
Janeiro und damit erstmalig in Brasilien statt und stellte mit 111 teilnehmenden

Ländern und 615 Schülerinnen und Schülern einen neuen Größenrekord auf.
\end{abstract}

Insgesamt 62 Mädchen nahmen in diesem Jahr teil. Ihr relativer Anteil von 10\% ist seit vielen Jahren fast konstant. Das deutsche Team bestand aus sechs Schülern, Eric Müller als stellvertretendem Delegationsleiter und dem Berichterstatter als Delegationsleiter.

Tabelle 1. Das deutsche Team

\begin{tabular}{lllc}
\hline Name & Wohnort & Schule & Klasse \\
\hline $\begin{array}{c}\text { Armbruster, } \\
\text { Alexander }\end{array}$ & Unterhaching & $\begin{array}{l}\text { Lise-Meitner-Gymnasium } \\
\text { Unterhaching }\end{array}$ & 11 \\
$\begin{array}{c}\text { Drees, } \\
\text { Martin }\end{array}$ & Cadolzburg & $\begin{array}{l}\text { Dürer-Gymnasium Nürn- } \\
\text { berg }\end{array}$ & 12 \\
$\begin{array}{c}\text { Juran, } \\
\text { Branko }\end{array}$ & Berlin & $\begin{array}{l}\text { Heinrich-Hertz- } \\
\text { Oberschule Berlin }\end{array}$ & 12 \\
$\begin{array}{c}\text { Meyer, } \\
\text { Sebastian }\end{array}$ & Dresden & $\begin{array}{l}\text { Martin-Andersen-Nexö- } \\
\text { Gymnasium Dresden }\end{array}$ & 12 \\
$\begin{array}{c}\text { Paul, } \\
\text { Manfred }\end{array}$ & Rimpar & $\begin{array}{l}\text { Deutschhaus-Gymnasium } \\
\text { Walter, }\end{array}$ & 12 \\
Jonas & Rostock & $\begin{array}{l}\text { Gymnarg } \\
\text { Gonium Reutershagen }\end{array}$ & 10 \\
\hline
\end{tabular}

Die vier Abiturienten hatten schon IMO-Erfahrung. 2016 in Hongkong gewannen Martin Drees und Sebastian Meyer eine Silber- und Branko Juran und Manfred Paul eine Bronzemedaille. Für Sebastian Meyer war es die inzwischen dritte IMO-Teilnahme; er erkämpfte sich 2015 in Thailand eine Bronzemedaille. Alle sechs Schüler können auf eine langjährige erfolgreiche Teilnahme an nationalen Mathematik-Wettbewerben zurückblicken.

\section{Auswahl und Vorbereitung der deutschen Mannschaft}

Für die Auswahl und Vorbereitung der deutschen Mannschaft wurde wie in den Vorjahren verfahren. Es qualifizierten sich 29 Schülerinnen und 137 Schüler durch die erfolgreiche Teilnahme an der Bundesrunde der Mathematik-Olympiade oder an der 2. Runde des Bundeswettbewerbs Mathematik für zwei Auswahlklausuren, die am 1. und 7. Dezember 2016 geschrieben wurden. Hieran nahmen 24 Schülerinnen und 116 Schüler teil.

Die 16 erfolgreichsten Klausurteilnehmer bildeten den Kandidatenkreis für das deutsche Team. Für diese 15 Schüler und eine Schülerin gab es Seminare über eine knappe Woche in Rostock, zwei verlängerte Wochenenden in Bad Homburg und die traditionelle Abschlusswoche am Mathematischen Forschungsinstitut in Oberwolfach. Der übliche dritte Wochenendlehrgang fand in diesem Jahr nicht in Bad Homburg, sondern direkt vor der Bundesrunde der Mathematik-Olympiade in Bremerhaven statt. Alle 16 Teilnehmer hatten sich für die Bundesrunde qualifiziert. Während dieser fünf Lehrgänge wurden von allen Kandidaten insgesamt sieben Klausuren geschrieben. Die sechs Besten qualifizierten sich für das IMO-Team, (s. Tabelle 1), dessen Zusammensetzung am 25. Mai in Oberwolfach offiziell bekannt gegeben wurde.

Zusätzlich gab es vom 16. bis 18 . Juni einen von Dierck Schleicher organisierten Lehrgang an der Jacobs University Bremen, traditionell am Samstag mit dem Turnier „MatBoj“. Schließlich organisierte das Team selbstständig ein Intensivtraining, dass vom 24. bis 27. Juni in einer Jugendherberge in Bonn stattfand. Wie in den Vorjahren berichteten die Teilnehmer von intensiver Beschäftigung mit selbst herausgesuchtem Aufgabenmaterial.

Zum wiederholten Mal fand ein Zusatztraining im Vorfeld dieses Auswahlverfahrens statt. Eric Müller, Jens Reinhold, Lisa Sauermann und Florian Schweiger betreuten in der zweiten Jahreshälfte 2016 per E-MailKorrespondenz sehr intensiv acht Schüler, die schon an IMO-Vorbereitungslehrgängen teilgenommen hatten und die auch noch in diesem Jahr startberechtigt waren. Sieben dieser acht Schüler qualifizierten sich über die beiden Dezemberklausuren wieder für die Vorbereitungslehrgänge und belegten dort am Ende die ersten sieben Plätze.

Seit 2007 gibt es das Programm Jugend trainiert Mathematik (JuMa). Es wurde unter anderem zur besseren Vorbereitung unserer Schülerinnen und Schüler auf die IMO initiiert. Viele der erfolgreichen Teilnehmer an den bundesweiten Mathematik-Wettbewerben und auch unsere sechs IMO-Teilnehmer wurden durch dieses Projekt gefördert. Leider stehen für das neue Schuljahr 2017/18 nötige Finanzmittel nicht wie bisher zur Verfügung. Seit längerer Zeit versuchen daher die für JuMa verantwortlichen Kollegen zusammen mit dem Mathematik-Olympiaden e. V. Lösungen zu finden, die die weitere Durchführung von JuMa ermöglichen.

Die gesamte organisatorische Vorbereitung und Durchführung der Klausuren, der Seminare und der Reise wurden wiederum von der Geschäftsstelle der Bundesweiten Mathematik-Wettbewerbe unter Leitung von HannsHeinrich Langmann in gewohnt perfekter Weise abge- 


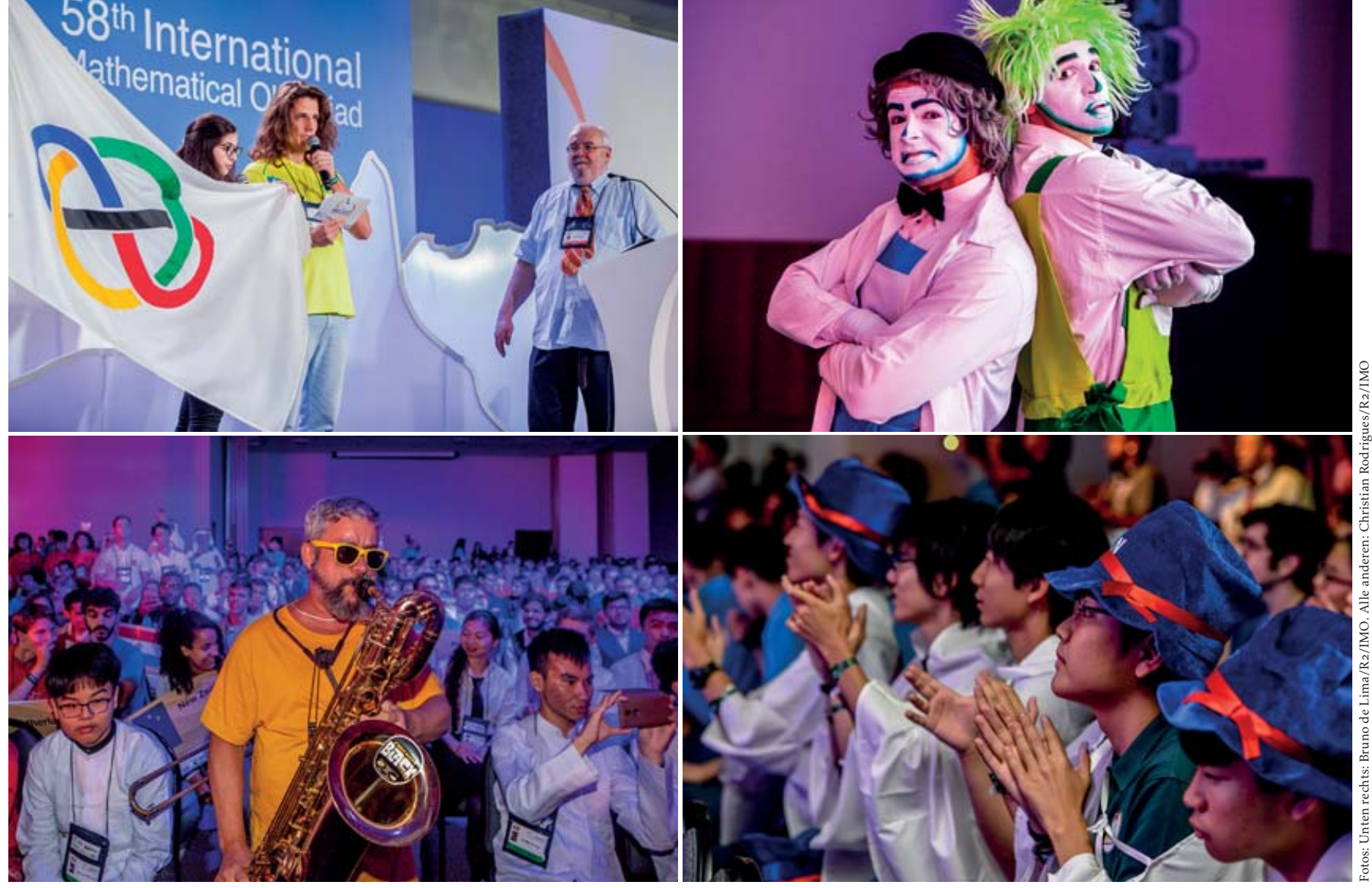

Eröffnungsfeier der 58. IMO im Hotel Windsor Oceanico

wickelt. Allen, die an der Organisation und der Vorbereitung des deutschen Teams beteiligt waren, gebührt herzlicher Dank.

\section{Der Ablauf der 58. IMO}

Die Delegationsleiter der Länder, welche die internationale Jury bilden, reisten am 12./13. Juli an.

Die Eröffnungsfeier fand am 17. Juli im Hotel Windsor Oceanico statt. Vor der Parade der 111 Mannschaften gab es eine Schweigeminute für die drei Tage zuvor verstorbene iranische Fields-Medaillen-Preisträgerin Maryam Mirzakhani. Der Direktor des Instituto Nacional de Matemática Pura e Aplicada, Marcelo Viana, fand sehr emotionale Worte für diese Ausnahmemathematikerin, die 1994 und 1995 an der IMO teilgenommen und mit 41 und 42 Punkten jeweils eine Goldmedaille gewonnen hatte.

Am 18. und 19. Juli wurden vormittags die beiden $4^{1 / 2-}$ stündigen Klausuren geschrieben. Zur Beantwortung der Verständnisfragen, die die Schüler in der ersten halben Stunde der Klausuren schriftlich stellen konnten, kamen die Delegationsleiter dieses Mal direkt zum Ort der Klausuren. Die schnelle Internetübertragung der Fragen und Antworten erschien den Veranstaltern zu unsicher. Außerdem gab es eine weitere echte Neuerung, die sicherstellen sollte, dass alle Teilnehmenden ihre Antworten innerhalb einer Stunde bekommen. Wenn, wie in den letzten Jahren, alle Antwortvorschläge erst von der gesamten Jury abgestimmt werden müssen, ist dies nicht zu schaffen. Erstmalig wurde daher pro Aufgabe ein Team von Koordi- natoren und freiwilligen Jurymitgliedern gebildet, die die Antwortvorschläge mit den jeweiligen Delegationsleitern diskutiert und dann genehmigt haben. Dieses neue Verfahren hat wesentlich zur schnelleren Beantwortung der Fragen beigetragen.

Nach der Durchsicht der Schülerlösungen durch die Delegationsleitungen fand vom 20. bis 21. Juli die endgültige Festlegung der Bewertung mit den Koordinatoren statt. Hierzu hatten die Veranstalter ein Team von 82 Experten zusammengestellt. Aus Deutschland war in dieser Funktion Stephan Neupert von der LMU München beteiligt.

Am Vormittag des letzten Tages hielt der FieldsMedaillen-Preisträger Artur Ávila die inzwischen zur Tradition gewordene „IMO Lecture“. Er spannte den Bogen von seiner eigenen IMO-Goldmedaille (IMO 1995 in Toronto) hin zu seiner jetzigen Forschung.

Die Preisverleihung fand am 22. Juli wieder im Hotelkomplex der Teams statt. Nach dem Dank an die Sponsoren wurden alle Preisträger in größeren Gruppen auf die Bühne gebeten. Zum Abschluss wurde die IMO-Fahne feierlich an den nächsten Veranstalter Rumänien übergeben und mit einem Film über Cluj-Napoca und die IMO 2018 für das nächste Jahr eingeladen. An die Siegerehrung schloss sich die traditionelle Farewell Party an, auf der auch wieder das "goldene Mikrofon“ an das Jury-Mitglied mit den meisten Redebeiträgen in den Jury-Sitzungen überreicht wurde. In diesem Jahr ging dieser inoffizielle Preis an den israelischen Delegationsleiter Dan Carmon. 


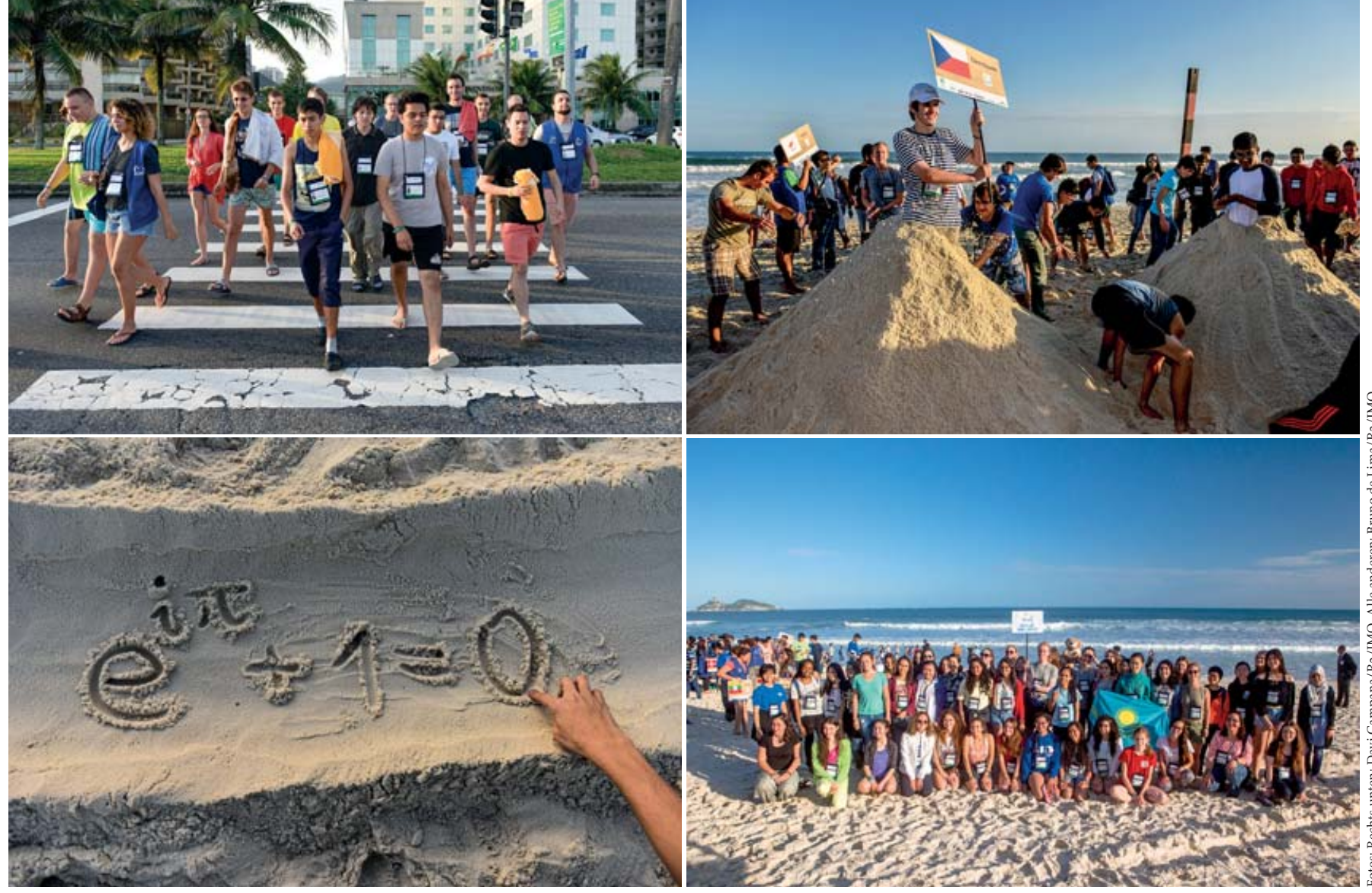

Erholung

Nachdem die Jury diese finale englische Version bestätigt hatte, wurden die Aufgaben in die vier anderen offiziellen Sprachen Deutsch, Französisch, Russisch und Spanisch übersetzt und nach einiger Diskussion, besonders zu den Aufgaben 3 und 5, ebenfalls von der Jury bestätigt. Jeder Schüler und jede Schülerin erhält die Aufgaben in der Muttersprache und einer zweiten Sprache eigener Wahl. Demgemäß übersetzten die entsprechenden Delegationsleiter die Aufgabentexte in die restlichen 53 Sprachen. Auch alle diese Versionen wurden nach Prüfung durch die Jury bestätigt. Ab dem nächsten Jahr sollen alle Schüler bis zu drei Sprachversionen erhalten, damit sichergestellt ist, dass sie mindestens eine der fünf offiziellen Sprachversionen vorliegen haben. Insgesamt standen die Aufgaben in 58 Sprachversionen zur Verfügung und sind auf www.imo-official.org verfügbar.

Bei der Bewertung der Lösungen wurden 34,7\% der möglichen Punkte vergeben. Diese IMO lag damit wie 2016 mit 35,2 \% gut im Durchschnitt der letzten zehn Jahre. Die beiden Extrema in diesen zehn Jahren waren 2014 mit $38,2 \%$ und 2015 mit 30,9\%.

Allerdings ergab sich bei der Verteilung der Punkte auf die sechs Aufgaben eine Besonderheit. Die beiden leichten Aufgaben wurden von vielen als wirklich (relativ) leicht angesehen, wohingegen sich die Aufgaben 2, 3, 5 und 6 als ausgesprochen schwer herausstellten. Tabelle 2 belegt dies sehr anschaulich. Die spannende Aufgabe 3 ist sogar die IMO-Aufgabe mit den wenigsten durchschnittlich erreichten Punkten überhaupt! Auch die diesjährige
Aufgabe 6 schafft es noch unter die Top 7. Aufgaben mit absolut nicht mehr als 26 erreichten Punkten gab es nur 1959-1963 und 1971. Es überrascht daher nicht, dass auf keiner der vorherigen 57 IMOs der beste Teilnehmer so wenig Punkte wie die drei besten Teilnehmer in diesem Jahr erreichte. Je ein Schüler aus dem Iran, Japan und Vietnam erreichte 35 Punkte.

In der Hall of Fame aller IMO-Teilnehmer seit 1959 (www.mathematik-olympiaden.de oder www.imo-official. org/hall.aspx) gab es an der Spitze keine Veränderungen. In dieser Liste liegen unverändert Lisa Sauermann auf Platz 3, Christian Reiher auf Platz 5, Wolfgang Burmeister auf Platz 7, Martin Härterich auf Platz 10 und Peter Scholze auf Platz 11. Auch im exklusiven Club der IMOTeilnehmer mit mindestens 3 Goldmedaillen gab es in diesem Jahr keine Neuaufnahmen.

Das Reglement sieht vor, dass nicht mehr als die Hälfte der Teilnehmer einen Preis erhält und dass die Anzahlen der 1., 2. bzw. 3. Preise möglichst das Verhältnis $1: 2: 3$ aufweisen sollten. Die diesjährigen Punktgrenzen sind in Tabelle 4 angegeben. Wieder wurde darüber anonym in der Jury abgestimmt. Anonym meint hier, dass der Jury gesagt wurde: „Wenn man für $n$ Punkte eine Medaille bekommt, dann werden insgesamt 318 Medaillen vergeben, bei Punktgrenze $n+1$ genau 291 Medaillen." Bei der Juryabstimmung, ob mehr als die laut Reglement möglichen 307 Teilnehmer eine Medaille bekommen sollen und bei der $n$ den Jurymitgliedern noch unbekannt war, wurde die Zweidrittelmehrheit zum Überstimmen 


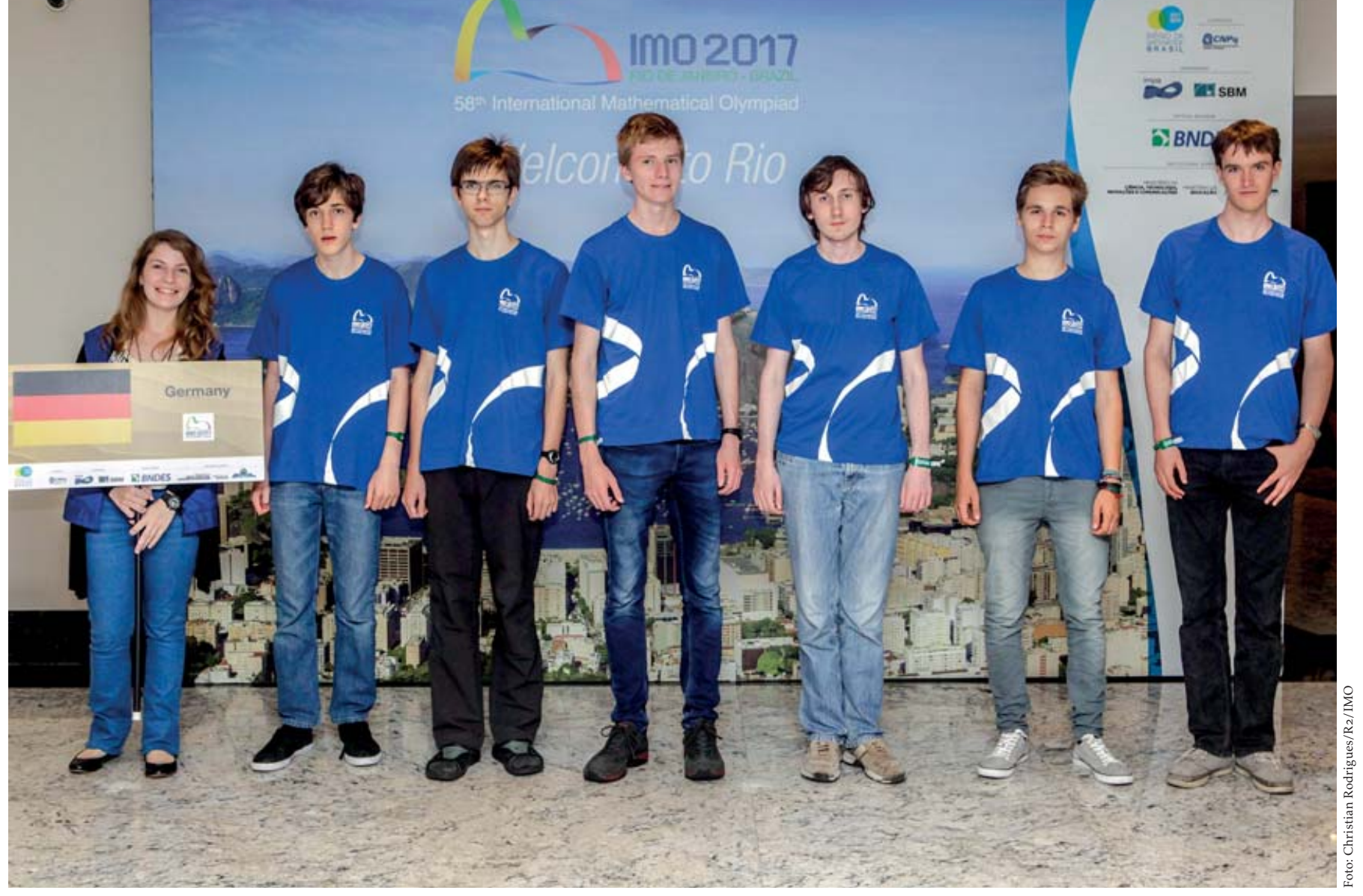

Das deutsche Team: Mylla Coffaro Ferreira (Guide), Jonas Walter, Sebastian Meyer, Martin Drees, Manfred Paul, Branko Juran, Alexander Armbruster (v.1.n.r.)

des Reglements knapp verfehlt. Analog wurde auch bei den Grenzen für Silber und Gold von der Jury anonym die jeweils höhere Punktgrenze ausgewählt. Es gab auch in diesem Jahr keinen Sonderpreis für die besonders elegante Lösung einer Aufgabe.

\section{Das deutsche Team}

Das Ergebnis des deutschen Teams findet man in Tabelle 5. Gefreut haben wir uns über die vier Medaillen. Manfred Paul und Sebastian Meyer haben die Bronzemedaille nur um einen Punkt verpasst. Auch Alexander Armbruster und Jonas Walter haben Gold beziehungsweise Silber jeweils um nur einen Punkt verfehlt. In der inoffiziellen Länderwertung liegen wir in diesem Jahr auf Rang 33, nach Rang 19 auf der letztjährigen IMO in Hongkong. In den Jahren davor erzielten wir 2015 in Thailand und 2013 in Kolumbien Rang 27 und 2014 in Südafrika Rang 16.

Die beiden Besten aus dem Team können sich wieder für die IMO 2018 qualifizieren, Jonas Walter kann sogar noch zweimal teilnehmen.

Häufig wird die Frage gestellt, in welchen Gebieten die deutschen Schüler besonders gut bzw. schlecht sind. In diesem Jahr mit zwei leichten, aber kaum mittelschweren Aufgaben ist Tabelle 2 dazu eher wenig aussagekräftig.
Ausblick

In diesem Jahr bestätigte die Jury den Veranstalter für 2022 (vgl. Tabelle 6). Eine Interessensbekundung aus Japan für eine IMO 2023 in Tokio liegt vor.

\section{Das IMO-Board}

In diesem Jahr beschloss die Jury einige kleinere Änderungen des Reglements. Dazu gehört die Umbenennung des IMO-Advisory-Boards und des Vorsitzenden in IMOBoard und Präsident. Turnusgemäß fanden keine Wahlen statt, sodass sich nur Änderungen bei den Ex-OfficioMitgliedern ergaben. Die neue Zusammensetzung dieses Gremiums ist in Tabelle 7 angegeben.

\section{Weitergehende Informationen über die IMO}

Speziell zu den IMOs sind die beiden folgenden Webseiten empfehlenswert:

www.imo-official.org

www.mathematik-olympiaden.de/IMOs/imo.html

Für weitere Informationen zu mathematischen Schülerwettbewerben sei auf die Webseite www. mathe-wettbewerbe.de hingewiesen. 
Tabelle 2. Die Ergebnisse der einzelnen Aufgaben

\begin{tabular}{clrrc}
\hline Aufgabe & Gebiet & $\begin{array}{c}\text { Alle } \\
(\%)\end{array}$ & $\begin{array}{c}\text { Top 10 } \\
(\%)\end{array}$ & $\begin{array}{r}\text { Deutsches Team } \\
(\%)\end{array}$ \\
\hline 1 & Zahlentheorie & 84,9 & 99,0 & 97,6 \\
2 & Algebra & 32,9 & 68,1 & 38,1 \\
3 & Kombinatorik & 0,6 & 1,4 & 0,0 \\
4 & Geometrie & 71,8 & 98,8 & 81,0 \\
5 & Kombinatorik & 13,8 & 42,6 & 35,7 \\
6 & Zahlentheorie & 4,2 & 30,5 & 0,0 \\
Alle & & 34,7 & 56,7 & 42,1 \\
\hline
\end{tabular}

Tabelle 3. Kleinste durchschnittlich erreichte Punktzahl seit der 1. IMO 1959

\begin{tabular}{crrr}
\hline$\varnothing$ & \multicolumn{1}{c}{ Absolute Punkte } & Aufgabe & Jahr \\
\hline 0,042 & $3 \times 1+1 \times 4+1 \times 5+2 \times 7=26$ & 3 & 2017 \\
0,152 & $40 \times 1+2 \times 2+5 \times 7=79$ & 6 & 2007 \\
0,168 & $2 \times 1+1 \times 2+10 \times 3+6 \times 4+2 \times 5+1 \times 6+3 \times 7=95$ & 6 & 2009 \\
0,187 & $11 \times 1+3 \times 2+3 \times 3+1 \times 5+1 \times 6+8 \times 7=93$ & 6 & 2006 \\
0,251 & $25 \times 1+14 \times 2+2 \times 5+3 \times 6+10 \times 7=151$ & 3 & 2016 \\
0,260 & $31 \times 1+9 \times 2+1 \times 6+12 \times 7=139$ & 6 & 2008 \\
0,294 & $24 \times 1+9 \times 2+5 \times 3+4 \times 4+2 \times 5+14 \times 7=181$ & 6 & 2017 \\
0,296 & $15 \times 1+6 \times 2+6 \times 3+2 \times 4+6 \times 5+4 \times 6+7 \times 7=156$ & 6 & 2013 \\
\hline
\end{tabular}

Tabelle 4. Die Punktgrenzen für die Preise

\begin{tabular}{rll}
\hline 48 & Goldmedaillen & für $\geq 25$ Punkte (von 42) \\
90 & Silbermedaillen & für $\geq 19$ Punkte \\
153 & Bronzemedaillen & für $\geq 16$ Punkte \\
291 & Medaillen & bei 615 Teilnehmern \\
\hline
\end{tabular}

Tabelle 5. Die Ergebnisse des deutschen Teams

\begin{tabular}{lcl}
\hline Name & Punkte & Preis \\
\hline Alexander Armbruster & 24 & Silber \\
Jonas Walter & 18 & Bronze \\
Branko Juran & 17 & Bronze \\
Martin Drees & 17 & Bronze \\
Manfred Paul & 15 & Ehrende Erwähnung \\
Sebastian Meyer & 15 & Ehrende Erwähnung \\
\hline
\end{tabular}

Tabelle 6. Die nächsten IMOs

\begin{tabular}{llll}
\hline Jahr & Land & Ort & Zeitraum \\
\hline 2018 & Rumänien & Cluj-Napoca & 03.-14. Juli 2018 \\
2019 & Vereinigtes Königreich & Bath & 11.-22. Juli 2019 \\
2020 & Russland & St. Petersburg & 07.-18. Juli 2020 \\
2021 & USA & & \\
2022 & Norwegen & & \\
\hline
\end{tabular}

Tabelle 7. Die Mitglieder des IMO-Boards

\begin{tabular}{lc}
\hline & Amtszeit \\
\hline $\begin{array}{l}\text { Präsident: Geoff Smith (Vereinigtes Königreich) } \\
\text { Sekretär: Gregor Dolinar (Slowenien) }\end{array}$ & bis 2018 \\
Mitglied: Nazar Agakhanov (Russland) & bis 2018 \\
$\begin{array}{l}\text { Mitglied: Dávid Kunszenti-Kovács (Norwegen) } \\
\text { Mitglied: Yongjin Song (Südkorea) }\end{array}$ & bis 2020 \\
$\begin{array}{l}\text { ex officio IMO 2017: Edmilson Luis Rodrigues Motta } \\
\text { (Brasilien) }\end{array}$ & bis 2018 \\
$\begin{array}{l}\text { ex officio IMO 2018: Radu Gologan (Rumänien) } \\
\text { ex officio IMO 2019: Geoff Smith (Vereinigtes König-- }\end{array}$ & bis 2019 \\
reich) & \\
\hline
\end{tabular}

Prof. Dr. Jürgen Prestin, Institut für Mathematik, Universität zu Lübeck, Ratzeburger Allee 160, 23562 Luebeck prestin@math.uni-luebeck.de

Jürgen Prestin ist seit 2000 Inhaber einer Professur für Mathematik an der Universität zu Lübeck. Seine Forschungsschwerpunkte liegen in Approximationstheorie und Fourier-Analysis. Seit 2010 ist er 1. Vorsitzender des Mathematik-Olympiaden e. V. und seit 2015 Delegationsleiter der deutschen IMO-Mannschaft. 


\section{Tag}

1. Für jede ganze Zahl $a_{0}>1$ sei die Folge $a_{0}, a_{1}, a_{2}, \ldots$ gegeben durch

$$
a_{n+1}=\left\{\begin{array}{ll}
\sqrt{a_{n}} & \text { falls } \sqrt{a_{n}} \text { ganzzahlig, } \\
a_{n}+3 & \text { sonst, }
\end{array} \text { für alle } n \geqslant 0 .\right.
$$

Man bestimme alle Werte von $a_{0}$, so dass es eine Zahl $A$ gibt, mit $a_{n}=A$ für unendlich viele Werte von $n$.

(Südafrika)

2. Es sei $\mathbb{R}$ die Menge der reellen Zahlen. Man bestimme alle Funktionen $f: \mathbb{R} \rightarrow \mathbb{R}$, so dass für alle reellen Zahlen $x$ und $y$ gilt

$$
f(f(x) f(y))+f(x+y)=f(x y)
$$

(Albanien)

3. Ein Jäger und ein unsichtbarer Hase spielen in der euklidischen Ebene ein Spiel. Der Ausgangspunkt $A_{0}$ des Hasen und der Ausgangspunkt $B_{0}$ des Jägers sind gleich. Nach $n-1$ Runden des Spiels befinden sich der Hase im Punkt $A_{n-1}$ und der Jäger im Punkt $B_{n-1}$. Die $n$-te Runde des Spiels besteht aus drei Schritten in der angegebenen Reihenfolge:

(i) Der Hase bewegt sich unsichtbar zu einem Punkt $A_{n}$, so dass der Abstand zwischen $A_{n-1}$ und $A_{n}$ genau eins ist.

(ii) Ein Ortungsgerät meldet dem Jäger einen Punkt $P_{n}$. Die einzige Garantie, die das Ortungsgerät dem Jäger gibt, ist, dass der Abstand zwischen $P_{n}$ und $A_{n}$ höchstens eins ist.

(iii) Der Jäger bewegt sich sichtbar zu einem Punkt $B_{n}$, so dass der Abstand zwischen $B_{n-1}$ und $B_{n}$ genau eins ist.

Ist es immer möglich, egal wie sich der Hase bewegt und egal welche Punkte das Ortungsgerät meldet, dass der Jäger seine Bewegungen so wählen kann, dass der Abstand zwischen ihm und dem Hasen nach $10^{9}$ Runden höchstens 100 ist?

(Österreich)
2. $\mathrm{Tag}$

4. Es seien $R$ und $S$ verschiedene Punkte auf einem Kreis $\Omega$, so dass $R S$ kein Durchmesser ist. Es sei $\ell$ die Tangente an $\Omega$ in $R$. Der Punkt $T$ liegt so, dass $S$ der Mittelpunkt der Strecke $R T$ ist. Ein Punkt $J$ ist auf dem kleineren Bogen $R S$ von $\Omega$ so gegeben, dass der Umkreis $\Gamma$ des Dreiecks JST die Gerade $\ell$ in zwei verschiedenen Punkten schneidet. Es sei $A$ derjenige gemeinsame Punkt von $\Gamma$ und $\ell$, der näher an $R$ liegt. Die Gerade $A J$ schneidet $\Omega$ in einem weiteren Punkt K.

Man beweise, dass die Gerade KT den Kreis $\Gamma$ berührt.

(Luxemburg)

5. Gegeben sei eine ganze Zahl $N \geqslant 2$. Eine Gruppe von $N(N+1)$ Fußballspielern, von denen keine zwei gleich groß sind, steht in einer Reihe. Pelé möchte $N(N-1)$ Spieler so aus dieser Reihe entfernen, dass eine neue Reihe von $2 \mathrm{~N}$ Spielern verbleibt, in der die folgenden $N$ Bedingungen gelten:

(1) Niemand steht zwischen den beiden größten Spielern.

(2) Niemand steht zwischen dem drittgrößten und dem viertgrößten Spieler.

(N) Niemand steht zwischen den beiden kleinsten Spielern. Man zeige, dass dies immer möglich ist.

(Russland)

6. Ein geordnetes Paar $(x, y)$ ganzer Zahlen heißt teilerfremder Gitterpunkt, wenn der größte gemeinsame Teiler von $x$ und $y$ eins ist. Für eine gegebene endliche Menge $S$ teilerfremder Gitterpunkte beweise man, dass es eine positive ganze Zahl $n$ und ganze Zahlen $a_{0}, a_{1}, \ldots, a_{n}$ gibt, so dass für alle $(x, y)$ in $S$ gilt:

$$
a_{0} x^{n}+a_{1} x^{n-1} y+a_{2} x^{n-2} y^{2}+\cdots+a_{n-1} x y^{n-1}+a_{n} y^{n}=1 .
$$

Arbeitszeit: $4^{1 / 2}$ Stunden an jedem Tag. Bei jeder Aufgabe waren 7 Punkte erreichbar. 


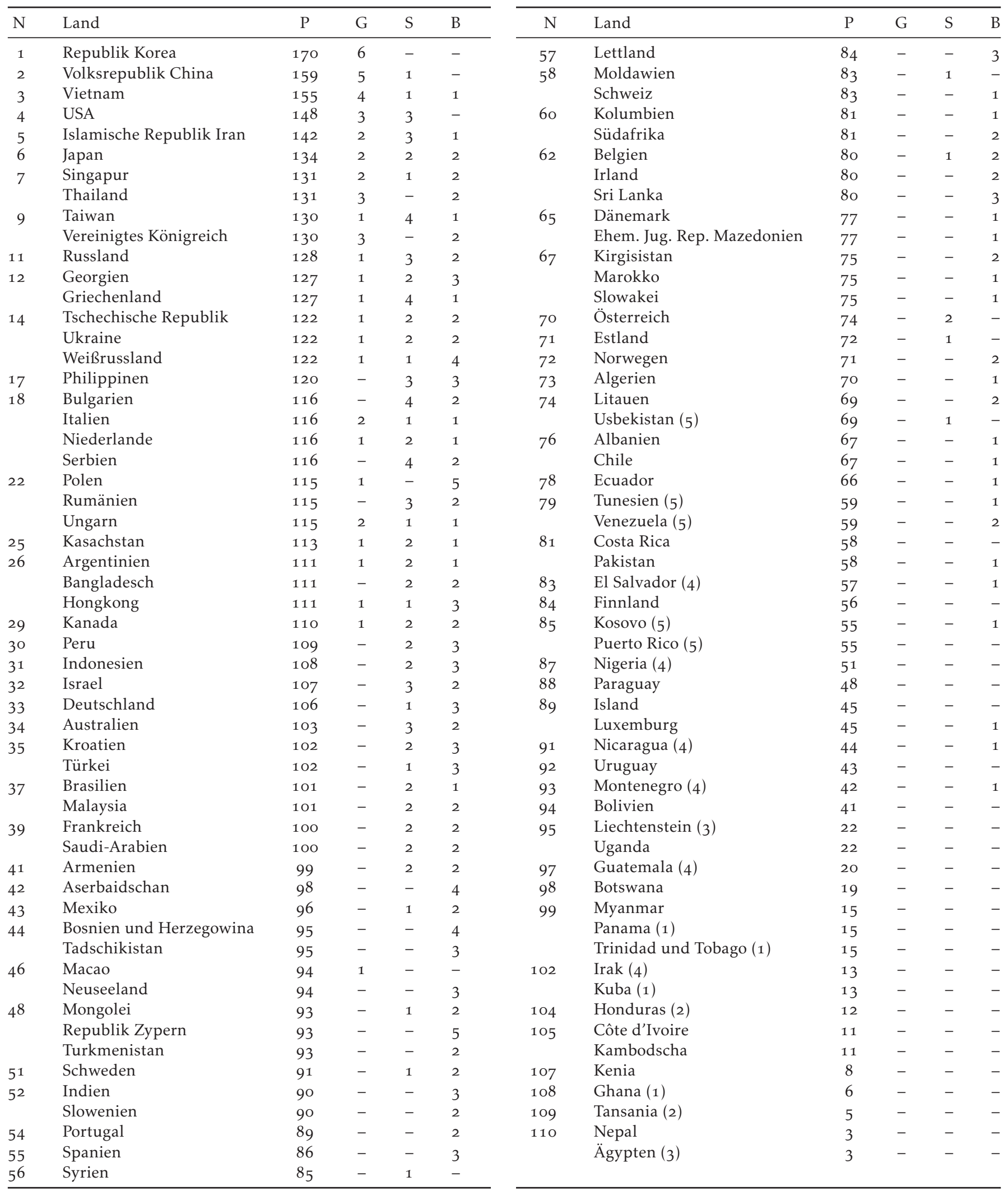

Legende: N - Platzierung, P - Punktzahl, G - Anzahl der Goldmedaillen, S - Anzahl der Silbermedaillen, B - Anzahl der Bronzemedaillen. Jede Mannschaft bestand aus sechs bzw. der in Klammern angegebenen Anzahl von Schülerinnen und Schülern. Eine vollständige Mannschaft (sechs Personen) konnte maximal 252 Punkte erreichen. 